\title{
Golimumab for the treatment of ulcerative colitis
}

\author{
Mathurin Flamant ${ }^{\mathrm{a}}$, Stephane Paul ${ }^{\mathrm{b}}$ and Xavier Roblin ${ }^{\mathrm{c}}$ \\ anstitut des Maladies de l'Appareil Digestif, Hotel Dieu, CHU de Nantes and Clinique Jules Verne, Nantes, France; 'baboratoire d'Immunologie et \\ Immunomonitoring, CIC 1408INSERM, GIMAP EA3064, Hôpital universitaire de Saint Etienne, Saint Priest en Jarez, France; 'CIC Inserm et Service \\ d'Hépato-gastroentérologie, Hôpital universitaire de Saint Etienne, Université Jean Monnet, Saint Priest en Jarez, France
}

\begin{abstract}
Introduction: Tumor necrosis factor antagonists have revolutionized the therapeutic management of inflammatory bowel disease. Infliximab and adalimumab were the first biological agents used to induce and maintain remission in ulcerative colitis. More recently, a third tumor necrosis factor antagonist, golimumab, was approved, extending the therapeutic approach for moderate-to-severe ulcerative colitis.

Areas covered: In this review, the authors review the literature on the efficacy and safety of golimumab in the context of other anti-TNF agents used in the treatment of this disease. The role of therapeutic drug monitoring in the case of loss of response to an anti-TNF agent is also discussed.

Expert opinion: Golimumab is currently effective to induce and maintain remission in patients with ulcerative colitis, especially those patients who are naive for an anti-TNF agent. No large studies have evaluated the efficacy of golimumab after failure of a first-line TNF antagonist therapy. In the case of loss of response to a first anti-TNF agent, therapeutic drug monitoring is essential to determine the most suitable therapeutic option.
\end{abstract}

ARTICLE HISTORY

Received 27 January 2017

Accepted 3 May 2017

\section{KEYWORDS}

Ulcerative colitis; TNF antagonist; golimumab

\section{Introduction}

Ulcerative colitis (UC) is a chronic inflammatory bowel disease (IBD) for which the incidence and prevalence are increasing globally [1]. The management of this disabling disease, which is characterized by symptoms of bloody diarrhea, abdominal pain, and asthenia, has been revolutionized during the last 15 years with the arrival of tumor necrosis factor (TNF) antagonists. TNF antagonists are used in patients who have not responded adequately to, or cannot use, conventional treatments. Over the last decade, infliximab and adalimumab have been the main biological therapies used for the treatment of UC, as they have been shown to induce clinical and endoscopic remission [2-4]. Golimumab, which is a TNF antagonist previously administered for rheumatic diseases such as rheumatoid arthritis, ankylosing spondylitis, and psoriatic arthritis, was approved for the treatment of UC by the FDA and EMA in 2013.

\section{Overview of the market}

Including golimumab, there are currently four biologics drugs approved for the management of UC in patients refractory to conventional therapy: three TNF antagonists (infliximab, adalimumab, and golimumab) and one anti-integrin (vedolizumab).

Infliximab was the first TNF antagonist approved to treat UC in 2006. ACT-1 and ACT-2 comprised a randomized, double-blind, placebo-controlled study that evaluated the efficacy and safety of infliximab for induction and maintenance therapy in UC patients (Table 1). In ACT-1, clinical response, clinical remission, and mucosal healing at week 54 were obtained with infliximab $(5 \mathrm{mg} / \mathrm{kg})$ in $45.5 \%, 34.7 \%$, and $45.5 \%$ of patients, respectively, compared with $19.8 \%, 16.5 \%$, and $18.2 \%$ of patients with placebo, respectively. In ACT-2, clinical response, clinical remission, and mucosal healing (week 30) were significantly higher with infliximab than with placebo $(47.1 \%, 25.6 \%, 46.3 \%$ and $26.0 \%, 10.6 \%, 30.1 \%$, respectively) [2].

Adalimumab was approved to treat UC in 2012. The two main differences of adalimumab compared with infliximab are the fully humanized nature of the TNF antagonist and a subcutaneous (SC) self-administration. Two large, randomized studies, ULTRA-1 and ULTRA-2, investigated the efficacy and safety of adalimumab in patients with moderately to severely active UC who were either naive for or previously exposed to TNF antagonists (Table 1). In these studies, patients receiving adalimumab were significantly more likely to be in clinical remission at weeks 8 and $52(16.5 \%$ and $17.3 \%$, respectively) compared with patients receiving placebo [4] (9.3\% and $8.5 \%$, respectively) $[3,4]$.

Vedolizumab is an a4 $\beta 7$ integrin inhibitor approved in 2014 for UC patients in the case of immunosuppressive therapy failure or TNF antagonist failure (EU and US indications differ). Vedolizumab blocks the interaction between an a4 $\beta 7$ integrin present on the surface of gut-specific lymphocytes and MAdCAM-1, a receptor on the vascular endothelium of the intestinal tract. In acting to disrupt leukocyte trafficking, vedolizumab has been proven to be effective, with approximately $40 \%$ sustained clinical remission in UC patients at week 52 [7]. 
Table 1. Clinical trials evaluating the efficacy of different anti-TNF agents in ulcerative colitis.

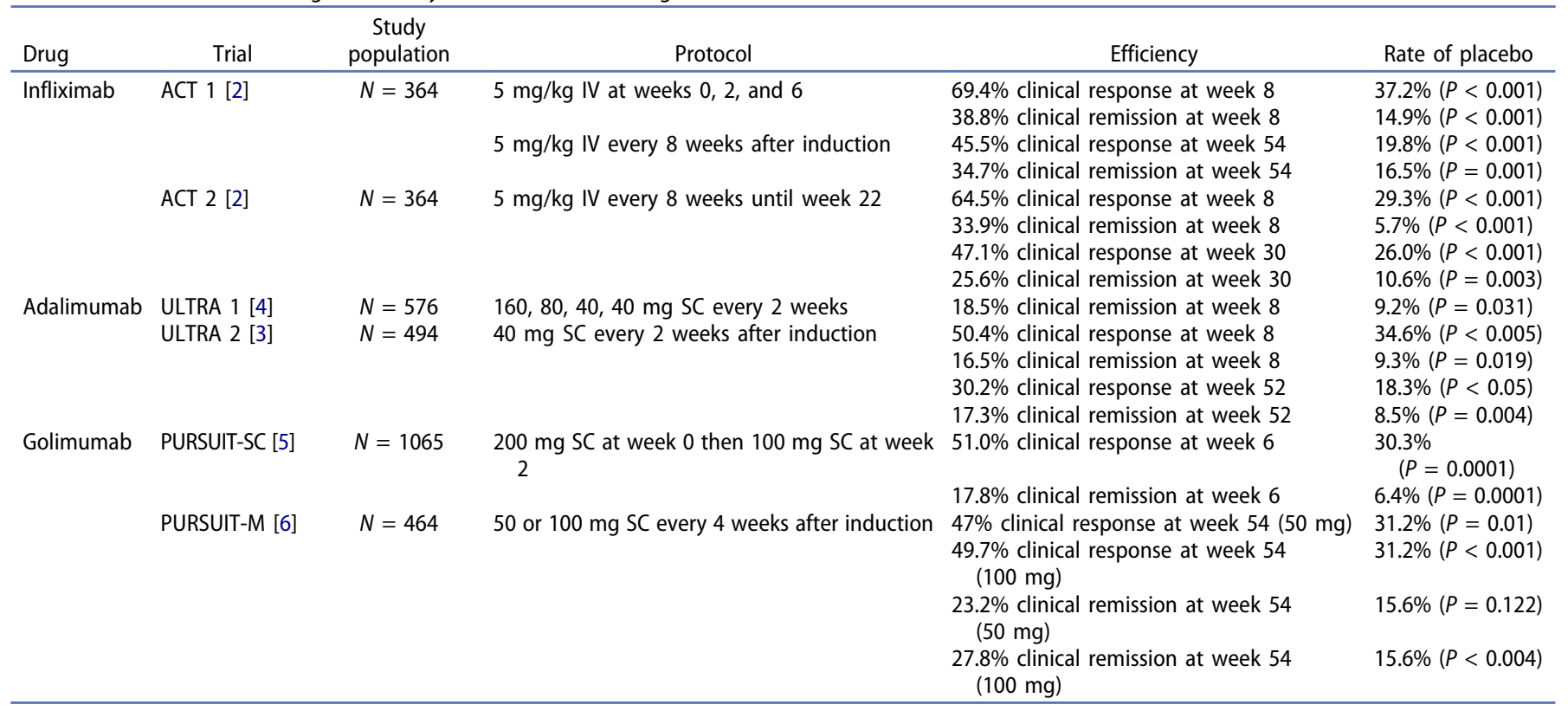

These results led to marketing authorization by the EMA for the treatment of adult patients with moderately to severely active UC who have had an inadequate response to, lost response to, or were intolerant to either conventional therapy or a TNF-alpha antagonist.

Among the molecules that are currently being developed for the treatment of patients with UC, another integrin inhibitor, etrolizumab, is being evaluated in a phase 3 study. Etrolizumab is a $\beta 7$-integrin inhibitor that prevents the interaction of integrins $\alpha 4 \beta 7$ and $a E \beta 7$ with their ligands, MAdCAM-1 and E-cadherin, respectively.

Similarly, small-molecule kinase inhibitors are promising therapies for IBD. Tofacitinib, which is already approved in rheumatology for the treatment of rheumatoid arthritis, has been successful in the treatment of IBD, with efficacy in inducing and maintaining clinical remission and mucosal healing in UC (OCTAVE Induction studies, NCT01465763; NCT 01458951). These two phase 3 studies were followed by an extension phase that was reported recently (OCTAVE Sustain, NCT01458574), showing that more patients receiving $5 \mathrm{mg}$ BID ( $N=198)$ and $10 \mathrm{mg}$ BID $(N=197)$ tofacitinib had achieved clinical remission $(34.3 \%$ and $40.6 \%$, respectively, vs. $11.1 \%$ placebo) and mucosal healing $(37.4 \%$ and $45.7 \%$, respectively, vs. $13.1 \%$ placebo) at week 52 . Filgotinib, another JAK inhibitor, is currently being evaluated in a phase 3 clinical trial for efficacy and safety in the induction and maintenance treatment of patients with moderate-to-severe UC (NCT02914522, estimated study completion date, December 2019; estimated enrollment, 1300 patients).

Finally, sphingosine 1-phosphate receptor modulator is a novel, selective small-molecule immunomodulatory agonist for G-protein-coupled S1P receptor (S1P1). Ozanimod (RPC1063) blocks lymphocyte egress from lymph nodes into the systemic circulation. A phase 3 study has been launched after the encouraging results of the phase 2 study (TOUCHSTONE) where clinical response, clinical remission and mucosal healing rates were statistically higher in patients receiving two different doses of ozanimod $(0.5$ and $1 \mathrm{mg}$ once daily) compared with placebo in two induction (week 8) and maintenance (week 32) phases. A recent review provides an update on the current status in clinical development of these new classes of therapeutics [8].

\section{Introduction to the compound}

Golimumab is a fully human monoclonal lgG1 antibody developed by Janssen Biotech, Inc. It is indicated 'for treatment of moderately to severely active UC in adult patients who have had an inadequate response to conventional therapy including corticosteroids and 6-mercaptopurine (6-MP) or azathioprine (AZA), or who are intolerant to or have medical contraindications for such therapies' (Box 1).

Elevated blood and tissue levels of the cytokine protein, TNF alpha, have been implicated in the pathophysiology of several chronic inflammatory diseases, such as rheumatic diseases (rheumatoid arthritis, psoriatic arthritis, ankylosing spondylitis) and intestinal diseases (Crohn's disease, UC). It has been shown that cytokines, chemokines, and growth factors play important roles in the pathophysiology of IBD in

\begin{tabular}{|c|c|}
\hline Drug name & Golimumab \\
\hline Phase & Approved \\
\hline Indication & Ulcerative colitis \\
\hline $\begin{array}{l}\text { Pharmacology } \\
\text { description }\end{array}$ & $\begin{array}{l}\text { Immunosuppressants, tumor necrosis factor alpha } \\
\text { (TNF-a) inhibitor }\end{array}$ \\
\hline $\begin{array}{l}\text { Route of } \\
\quad \text { administration }\end{array}$ & Subcutaneous \\
\hline Pivotal trial(s) & {$[5,6]$} \\
\hline
\end{tabular}


mediating inflammation, cell differentiation, and the migration of immune cells in inflamed tissues. Although TNF antagonists are known to promote a rapid reduction in the number of cells at the inflammatory site, the mechanism of action via which this occurs is unclear. The most obvious function of the TNF antagonists is an inactivation of the pro-inflammatory cytokine TNF by direct neutralization. TNF alpha exists in two biologically active forms: a transmembrane precursor at the surface of immune cells and a soluble form. Golimumab, like other TNF antagonists, acts by binding directly to both the soluble and transmembrane precursor forms. However, preclinical studies have shown that golimumab has a higher binding affinity than either infliximab or adalimumab for both soluble and transmembrane TNF alpha. In preventing the binding of TNF alpha to its receptors, golimumab inhibits the biological activity of TNF alpha. Indeed, two distinct receptors for TNF alpha are known (TNFR1 and TNFR2), and activation of these receptors by TNF alpha induces an intracellular signaling cascade, with effects involving cytokine secretion, cell proliferation, and apoptosis. In particular, activation of TNFR1 (especially with soluble TNF) activates nuclear factor kappa B receptor (NFKB), which translocates to the nucleus and activates the transcription of several pro-inflammatory cytokine genes, such as IL-8, IL-1, IL-6, COX-2, and TNF alpha.

It is likely that TNF antagonists exert several complex effects beyond simple TNF alpha neutralization, such as modulation of the immune system, actions on intestinal barrier function, and induction of immune cell apoptosis. TNF antagonists could then play a role by inducing regulatory $T$ cells (enhancement of anti-inflammatory T-helper cells), contributing to the resolution of inflammation. Moreover, TNF antagonists could modulate the expression of adhesion proteins responsible for leukocyte infiltration, such as E-selectin, ICAM-1, and VCAM-1, which can be involved in the inflammatory reaction via a role in leukocyte adhesion to the vascular endothelium. In the same way, many studies have evaluated the potential role of TNF antagonists in the programed cell death of immune cells induced by the Fc region of the antiTNF antibodies. However, all of these complex mechanisms contributing to the resolution of inflammation are still being debated and investigated.

\section{Pharmacology and metabolism}

Golimumab is available as a $0.5-\mathrm{ml}$ (50 mg golimumab) or $1-\mathrm{ml}$ (100 mg golimumab) solution, in a single-dose prefilled syringe or in a single-dose prefilled SmartJect autoinjector. The standard golimumab dose for treatment of UC is $200 \mathrm{mg}$ initially, administered by SC injection at week 0 , followed by $100 \mathrm{mg}$ at week 2, and then $50 \mathrm{mg}$ every 4 weeks (Q4) for a body weight less than $80 \mathrm{~kg}$ or $100 \mathrm{mg}$ Q4 for a body weight over $80 \mathrm{~kg}$ (EU), or $100 \mathrm{mg}$ (USA) every 4 weeks. The 4-week dosing interval for golimumab may be explained by an affinity of golimumab for soluble human TNF-alpha in vitro and in vivo that is significantly higher than that of adalimumab [9].

Following SC administration of $50 \mathrm{mg}$ golimumab, the median time to reach maximum serum concentration $\left(T_{\max }\right)$ ranges from 2 to 6 days, with a mean concentration $\left(C_{\max }\right)$ of approximately $3.1 \pm 1.4 \mu \mathrm{g} / \mathrm{ml}$. The absolute bioavailability via
SC administration is estimated to be approximately $53 \%$. The median half-life is estimated to be approximately 2 weeks [10].

The metabolism and route of elimination of golimumab are yet to be determined.

Across the studies detailed below, antibodies to golimumab were detected enzymatically using a drug-sensitive assay (EIA) in 3\% (26/946) of golimumab-treated patients (phase II and III UC studies through week 54). Sixty-eight percent (21/ 31) of antibody-positive patients had neutralizing antibodies in vitro. In these studies, concomitant immunomodulators (AZA, 6-MP, and methotrexate) resulted in a lower proportion of patients having antibodies to golimumab than for patients receiving golimumab monotherapy $[1 \%$ (4/308) vs. 3\% (22/ 638), respectively]. Antibodies to golimumab were detected in $4 \%(23 / 604)$ of golimumab-treated patients who continued in an extension of the study and had evaluable samples through week 228. Eighty-two percent (18/22) of antibodypositive patients had neutralizing antibodies in vitro [11].

\section{Clinical efficacy}

Studies leading to the approval of golimumab for UC, named the 'Program of Ulcerative Colitis Research Studies Utilizing an Investigational Treatment' (PURSUIT), were divided into two phases: induction (PURSUIT-SC) and maintenance (PURSUITM) $[5,6]$.

The first phase (PURSUIT-SC) was a multicenter, randomized, placebo-controlled induction study conducted between July 2007 and November 2010. This phase comprised a dose-finding phase (phase 2) and a dose-confirmation phase (phase 3). All patients included were treated for UC, with moderate-to-severe disease activity defined as a Mayo score of 6-12 with an endoscopic subscore $\geq 2$. All active patients were eligible, even those receiving only mesalazine. About one-third of patients received an immunomodulatory drug concomitantly. Unlike other pivotal studies for the treatment of UC, patients in this study were naive for TNF antagonists and other biological therapies. Previous pivotal trials of biological therapies have demonstrated that the efficacy of a biological agent is superior in patients naive for TNF antagonist compared with those who have already experienced a TNF antagonist [12]. At the present time, no large studies are evaluating the efficacy of golimumab after failure of a firstline TNF antagonist therapy.

In the phase 2 study, 169 patients were randomized to receive either placebo or different regimens of golimumab at week 0 and week 2: 100/50, 200/100, 400/200 mg. After analysis of the dose-finding data, the 400/200- and 200/100mg regimens were selected for the phase 3 study (774 patients).

In this phase $2 / 3$ induction study, golimumab treatment was associated with a higher level of clinical response, clinical remission, mucosal healing, and improved quality of life, as compared with placebo (Table 1). Indeed, the primary end point, clinical response at week 6 (defined as a decrease from baseline in the Mayo score $\geq 30 \%$ and $\geq 3$ points, accompanied by either a rectal bleeding subscore of 0 or 1 or a decrease from baseline in the bleeding subscore $\geq 1$ ), was achieved by $54.9 \%$ and $51.0 \%$ of patients receiving $400 /$ 
200 and 200/100 mg golimumab, respectively, compared with $30.3 \%$ of patients receiving placebo $(P<0.0001$ for both comparisons). In the same way, clinical remission at week 6 (a major secondary end point defined as a Mayo score $\leq 2$ points with no individual subscore $>1$ ) was achieved in $17.9 \%$ and $17.8 \%$ of patients receiving the two golimumab regimens (400/200 and 200/100 mg) compared with $6.4 \%$ of patients receiving placebo $(P<0.0001$ for both comparisons); mucosal healing (a major secondary end point defined as a Mayo endoscopic subscore of 0 or 1) was observed in $45.1 \%$ and $42.3 \%$ of patients receiving $400 / 200$ and $200 / 100 \mathrm{mg}$ golimumab, respectively, and $28.7 \%$ of patients receiving placebo $(P<0.0001$ and $P=0.0014$ for each comparison). The mean changes from baseline in inflammatory bowel disease questionnaire (IBDQ) score were 26.9 points in the $400 / 200-\mathrm{mg}$ group, 27.0 points in the 200/100-mg group, and 14.8 points in the placebo group ( $P<0.0001$ for both golimumab groups vs. placebo).

The PURSUIT program has also evaluated the efficacy of intravenous induction with golimumab in patients with moderate-to-severe UC (PURSUIT-IV), but the proportion of patients achieving clinical response was low and studies with intravenous golimumab administration were not subsequently continued [13].

The maintenance study (PURSUIT-M) was a multicenter (251 centers), randomized, placebo-controlled study conducted between September 2007 and October 2011. All patients who responded to golimumab induction therapy $(n=464)$ were randomized to receive placebo, $50 \mathrm{mg}$, or $100 \mathrm{mg}$ golimumab subcutaneously every 4 weeks (Q4W) through week 52.

The primary end point was a maintained clinical response among golimumab induction responders. This was achieved in $47 \%$ (50 mg) and 49.7\% (100 mg) of patients receiving golimumab, and $31.2 \%$ of patients receiving placebo $(P=0.010$ and $P<0.001$ vs. placebo, respectively). At week 54, a higher percentage of patients receiving 100-mg golimumab were in clinical remission (27.8\%) and had mucosal healing $(42.4 \%)$ than patients receiving placebo $(15.6 \%$ and $26.6 \%, P=0.004$ and $P=0.002$, respectively) or $50-\mathrm{mg}$ golimumab $(23.2 \%$ and $41.7 \%$, respectively) (Table 1 ).

A post hoc analysis of the PURSUIT studies aimed to determine longer term outcomes in patients with a delayed early response to golimumab therapy (response at week 14 among patients with absence of response at week 6) [14]. For these patients, clinical remission was obtained in 35.7\% (week 30) and $30.4 \%$ (week 54), similar to the rates in patients who were initially responders at week 6 (39.7\% at week 30 and $33.8 \%$ at week 54). Similarly, mucosal healing was obtained in $52.7 \%$ (week 30) and $42.9 \%$ (week 54) of week-14 responders compared with $56.3 \%$ (week 30 ) and $46.4 \%$ (week 54) of week-6 responders. It may then, in some cases, be important to wait for up to 14 weeks for a response to golimumab. In the absence of a response after 14 weeks, the treatment can be discontinued as efficacy is unlikely.

The efficacy of golimumab during 2 years of maintenance therapy was evaluated in 195 patients who were randomized to golimumab in PURSUIT-M. Efficacy was assessed by a physician's global assessment (PGA) every 3 months through week
104 , and an IBDQ every 6 months. Based on these two evaluation criteria, $86 \%$ of patients had inactive or mild disease activity at week 104. Among 174 patients who were corticosteroid free at week 54, 88.5\% remained corticosteroid free [15]. The main limitation of this study was that patients who participated in the long-term evaluation were initially responders to the induction therapy with golimumab and tolerant to the 52-week maintenance trial. Moreover, the efficacy was only evaluated by the PGA (a clinician's impression of the patient's disease status), which correlates poorly with endoscopic activity [16].

Several studies $[17,18]$ have attempted to compare the relative efficacies of different anti-TNF agents in the induction and maintenance of clinical response and remission in patients with UC. Most of these studies observed a similar efficacy of all anti-TNF agents, particularly in subjects being treated for the first time with a biological therapeutic agent. One meta-analysis reported that all biological agents (infliximab, adalimumab, golimumab, and vedolizumab) are effective treatments for UC, with adverse event rates similar to placebo. However, despite the fact that biologics have been widely investigated and used for a decade, no head-to-head trials have been realized [19].

In the case of loss of response, the PURSUIT-M study failed to define an optimization strategy. Indeed, in this study, nonresponder patients who initially received $50 \mathrm{mg}$ golimumab Q4W were re-randomized for a dose adjustment with either $50 \mathrm{mg}$ golimumab Q4W or $100 \mathrm{mg}$ golimumab Q4W. Among the 51 patients eligible for the dose adjustment, 26 patients continued to receive the 50-mg dose and 25 patients received a doubled dose of $100 \mathrm{mg}$. Comparing these two strategies, clinical response at week 54 did not differ significantly in these two groups $(28.0 \%$ for $100 \mathrm{mg}$ vs. $34.6 \%$ for $50 \mathrm{mg}$ ). Unfortunately, conclusions about a higher dose of golimumab cannot be drawn as, in the case of loss of response to $100 \mathrm{mg}$, although a dose adjustment to $200 \mathrm{mg}$ Q4W had been planned, this was not implemented due to a protocol amendment.

Pharmacokinetic measurements are being more frequently employed in the management of UC to predict clinical outcome in the induction and maintenance phases. It is interesting that measurement of golimumab concentration appeared to be useful in the maintenance study (PURSUIT-M), as a maintained clinical response at week 54 and clinical remission at weeks 30 and 54 were observed preferentially in patients with higher compared with lower serum golimumab concentration quartiles at week 54. Patients with the lowest golimumab concentration quartile were more likely to have factors known to be associated with higher clearance of golimumab, i.e. low albumin level, higher body weight, higher inflammatory burden (higher concentrations of CRP and fecal markers). Based on receiver operating characteristic curve analysis, a serum golimumab concentration of $2.5 \mu \mathrm{g} / \mathrm{ml}$ at week 6 and $1.4 \mu \mathrm{g} / \mathrm{ml}$ at week 44 seem to be an adequate concentration for induction and maintenance of clinical response. The percentage of patients with anti-golimumab antibodies was lower in the group who received concomitant immunosuppressive therapy compared with those who did not receive concomitant immunomodulators $(1.1 \%$ vs. $3.8 \%, P=0.013)[5,20]$.

A recent 'real-life' observational study confirmed that pharmacokinetic measurement of golimumab concentrations 
could predict clinical response in UC patients treated with this TNF antagonist. In 21 patients with moderate-to-severe UC, the median serum golimumab concentration was significantly higher in clinical responders than nonresponders at week 2 (10.0 vs. $7.4 \mu \mathrm{g} / \mathrm{ml}$, respectively; $P=0.035$ ) and week 6 (5.1 vs. $2.1 \mu \mathrm{g} / \mathrm{ml}$, respectively; $P=0.037$ ) [21]. However, although an association between clinical response and serum golimumab concentration has been shown, no prospective studies have demonstrated that adjustment of dosing based on serum levels provides superior clinical control.

As observed with previous TNF antagonists, some patients do not respond to golimumab induction therapy (primary nonresponders) or lose clinical remission over time (secondary nonresponders). In clinical practice, in these situations of loss of response, pharmacokinetic parameters based on anti-TNF trough levels and antidrug antibody concentrations could be helpful in the evaluation of patients treated with TNF antagonist therapies. In the PURSUIT studies, a significant link has been observed between clinical response and serum golimumab concentration. In the PURSUIT-SC and PURSUIT-M studies, patients with the higher golimumab serum concentration quartile had higher clinical response and remission rates compared with those in the lower serum concentration quartile. Based on these pharmacokinetic considerations, optimization of golimumab to the 100-mg dose could be considered in the case of loss of response to 50-mg golimumab, with the aim to obtain serum golimumab concentrations of 2.5 and $1.4 \mu \mathrm{g} / \mathrm{ml}$ in the induction and maintenance phases, respectively [20].

Interestingly, an ongoing prospective study from the German Inflammatory Bowel Disease Study group (enrollment started in October 2014) aims to optimize golimumab administration by calculating the probability of clinical response at week 26 based on pharmacokinetic data from week 6 . The primary outcome of this study is the prediction of response at week 26 based on a positive golimumab trough level $>2.5 \mu \mathrm{g} / \mathrm{ml}$ at week 6 and a $50 \%$ reduction in fecal calprotectin. This 'early MOnitoring of REsponse' (MORE) study should achieve a more thorough understanding of therapeutic development in patients with moderateto-severe UC, receiving regular doses of golimumab [22].

Lastly, golimumab is the most recently approved TNF antagonist for UC treatment and thus does not have a long prescription history. Long-term observational studies appear useful to evaluate the efficacy of the drug in a real-life setting. Hereupon, a large, prospective, observational European cohort study of patientreported continuous clinical response to golimumab in adults with moderately to severely active UC (GO OBSERVE) will start in early 2017 and will be conducted in 11 countries in Europe.

\section{Safety and tolerability}

In the PURSUIT induction and maintenance studies, golimumab therapy was safe and well tolerated, with an adverse event profile similar to that of placebo. Injection-site reactions were rare, erythema being the most frequently reported adverse event. In the maintenance study, among 464 patients, serious adverse events were reported in $7.7 \%, 8.4 \%$, and $14.3 \%$ receiving placebo, $50 \mathrm{mg}$, and $100 \mathrm{mg}$ golimumab, respectively. Four cases of tuberculosis were observed, including one fatal case. Two patients developed serious opportunistic infections (cytomegalovirus detected in the blood, Staphylococcus aureus and Nocardia cultured from a brain abscess), and three malignancies were reported (rectal, thyroid, and lung adenocarcinomas) [6]. In the study evaluating golimumab efficacy through two years of maintenance, the overall safety profile was similar compared with that observed at week 54. Interestingly, the serious adverse events occurred only in patients receiving $100 \mathrm{mg}$ golimumab [15].

The overall safety of golimumab has been best evaluated in the analysis of pooled data from long-term extension clinical trials (up to 5 years) in rheumatology, including patients with rheumatoid arthritis, psoriatic arthritis, and ankylosing spondylitis. Across 5 phase 3 studies, 2228 patients treated with SC golimumab were compared with 639 patients receiving placebo. The incidence of serious infections was similar with golimumab (3.29/100 patient-years) and placebo (4.86/100 patient-years) and did not appear to increase with the duration of golimumab therapy. In the same way, the overall timeadjusted incidence of malignancy was not higher with golimumab than placebo (1.07 vs. 2.59 per 100 patient-years). By contrast, higher tuberculosis, lymphoma, and demyelination rates were observed with golimumab versus placebo, although this risk level appeared to be higher only in patients receiving $100 \mathrm{mg}$ golimumab [23].

As with other TNF antagonists, it is recommended that patients with UC beginning treatment with golimumab respect standard precautions regarding the risk of infections. Screening for viral hepatitis and HIV is recommended, as well as for tuberculosis infection, which is crucial due to the risk of reactivation of a latent tuberculosis infection. A full medical history should be taken, and patients should undergo a physical examination, chest radiograph, and tuberculin skin test or IFN- $\gamma$ release assay. Moreover, in addition to adherence to routine vaccination schedules, specific recommendations for patients treated with TNF antagonists are advisable: patients who cannot provide a clear history of chickenpox illness should have serologic testing for varicella virus and should receive varicella vaccine if they do not show immunity. Similarly, other preemptive vaccinations are recommended, such as influenza, pneumococcal, or hepatitis B vaccinations. Current guidelines recommend that human papilloma virus vaccine should also be considered for all females between the ages of 9 and 26 years [24].

The risks associated with using golimumab during pregnancy have been evaluated in studies with the other anti-TNF agents. It is currently recommended to perform a risk-benefit balance evaluation for the use of a TNF antagonist during pregnancy, although these agents are considered to be relatively safe in this respect. TNF antagonists are high-molecular weight substances and placental transfer starts during the second trimester, after the 20th week of gestation [25]. A large study demonstrated a moderate risk of major birth defects in the prenatally exposed compared with nonexposed anti-TNF agent group ( $5 \%$ vs. $1.5 \%$, OR: 2.2 ) but no increased risk of malformations or spontaneous abortion [26]. In conclusion, in clinical practice, considering the impact of insufficiently controlled IBD on the mother and the unborn child, anti-TNF agent may be a treatment option for pregnant women with severe UC. 


\section{Regulatory affairs}

Golimumab was first approved in rheumatology, for rheumatoid arthritis, ankylosing spondylitis, and psoriatic arthritis (October 2009). It was approved by the FDA and EMA for the treatment of UC in September 2013.

\section{Conclusion}

In summary, including golimumab, three TNF antagonists are currently effective to induce and maintain remission in patients with UC. When indicated, the choice of which of these three treatments are selected can be discussed with the patient, and this may depend on the route of administration and patient preference. However, the concept of personalized medicine is emerging and ongoing studies aim to predict the clinical response of treatments based on pharmacokinetic parameters in the induction phase. Indeed, it is likely that therapeutic drug monitoring will soon be essential to determine the most suitable therapy. This would seem to be useful, as some patients do not respond to TNF antagonist treatment and may then benefit from other novel drugs, such as anti-integrin therapies. In the same way, other molecules that are being developed, such as JAK inhibitors, are promising and could enhance the therapeutic approach to UC in the coming years.

\section{Expert opinion}

Several studies have observed a similar efficacy of all effective anti-TNF agents in patients with UC, particularly in subjects being treated for the first time with a biological therapeutic agent. In pivotal clinical trials, as for the other anti-TNF agents, golimumab appears to be effective (Table 1). However, although all patients in the PURSUIT studies were naive for anti-TNF agents, the rates of clinical remission at weeks 6 and 54 were not higher than with the other anti-TNF agents.

Although no head-to-head studies have been realized to compare the real efficacies of biological therapies, an indirect comparison of infliximab versus adalimumab or golimumab has been published recently and proved that in the induction phase, infliximab and golimumab had similar efficacies and were more effective than adalimumab. In the maintenance phase, no differences were observed in efficacy between these three anti-TNF antagonists [27]. Another meta-analysis demonstrated comparable efficacy of infliximab and golimumab, superior to that of adalimumab, for achieving a sustained clinical response [28].

However, although golimumab, as for the other anti-TNF agents, is indicated for the treatment of moderately to severely active UC, its role in treating acute severe colitis remains to be determined. Indeed, in this case, intravenous infliximab appears to be the most effective and thus suitable therapy, due to its rapid action. Conversely, the application of golimumab by monthly SC injection has advantages over infliximab, which is administered intravenously, and adalimumab, which is administered at a 2-week dosing interval. Route of administration and patient preference may lead the choice of TNF antagonist.
Due to its recent approval and the lack of large studies, some questions are still debated in the management of UC with golimumab. First, in the induction therapy, the use of golimumab in association with another immunomodulatory drug is not clear. Indeed, unlike the SUCCESS study with infliximab in UC patients, where infliximab in combination with azathioprine was demonstrated to be superior to TNF antagonist monotherapy (patients were naive to both agents), in the PURSUIT study, a subanalysis failed to demonstrate superiority of combination therapy over golimumab monotherapy to maintain remission (about one-third of patients received another immunomodulatory drug concomitantly with golimumab treatment) [8]. Therefore, at present, we cannot recommend the systematic use of golimumab with an immunosuppressor, and other larger studies should be carried out to consider the best option for the induction therapy.

Second, in the case of loss of response with TNF antagonist treatment, different options are available to attempt to obtain a response. Indeed, based on data from other TNF antagonist treatments (not golimumab), therapeutic drug monitoring seems to be an essential component in determining the most suitable therapy, alongside optimization of the therapy with an increased dose of the drug, association with a concomitant immunomodulatory drug, or a switch to another therapeutic class. Bendtzen et al. have designed an algorithm which, although not yet validated in clinical studies, may prove useful in dealing with these complexities [29]. In particular, in this algorithm, for patients exhibiting a low drug concentration without antibodies against the drug, an increase in the dose is advised in theory. However, no studies have yet evaluated the efficacy and safety of golimumab for the treatment of UC at doses higher than $100 \mathrm{mg}$ Q4W. In rheumatoid arthritis only, in association with methotrexate, a randomized phase 2 study demonstrated an efficacy of adjustment of the golimumab dose regimen to $100 \mathrm{mg}$ Q2W as compared with $100 \mathrm{mg}$ Q4W golimumab, without increasing the risk of adverse events [30]. In the algorithm, the patients exhibiting a low drug concentration due to a high level of antibodies against the drug can, in theory, benefit from changing to another TNF antagonist treatment against which the patient has not yet developed antibodies. Association with an immunomodulatory drug also seems to be a good option. Indeed, based on previous infliximab and adalimumab studies, we could consider that adding a concomitant immunosuppressive therapy for patients treated with golimumab monotherapy has a possible impact on antidrug antibody formation, and hence on a beneficial clinical outcome [31,32]. Moreover, it has been demonstrated in several clinical trials that the risk of serious adverse events with combination therapy is not greater than that with TNF antagonist monotherapy $[33,34]$. Finally, in the algorithm, for patients with a high drug concentration, a switch to another therapeutic class should be considered. In particular, an a $4 \beta 7$ integrin antagonist acting via a different mechanism of action, inhibiting lymphocyte migration to the gastrointestinal mucosa by antagonism of the a4 $\beta 7$ gastrointestinal integrin receptor, has recently been approved for moderate-to-severe UC. With approximately $40 \%$ of sustained clinical remission in UC 
patients (GEMINI study, at week 52), vedolizumab has been proven to be effective and represents an alternative to the TNF antagonist strategy for UC patients in this situation.

\section{Funding}

This manuscript has not been funded.

\section{Declaration of interest}

The authors have no relevant affiliations or financial involvement with any organization or entity with a financial interest in or financial conflict with the subject matter or materials discussed in the manuscript. This includes employment, consultancies, honoraria, stock ownership or options, expert testimony, grants or patents received or pending, or royalties.

\section{References}

Papers of special note have been highlighted as either of interest $(\cdot)$ or of considerable interest $(\cdot \cdot)$ to readers.

1. Molodecky NA, Soon IS, Rabi DM, et al. Increasing incidence and prevalence of the inflammatory bowel diseases with time. Based Systematic Review Gastroenterology. 2012;1421:46-54 e42. quiz e30.

2. Rutgeerts P, Sandborn WJ, Feagan BG, et al. Infliximab for induction and maintenance therapy for ulcerative colitis. $\mathrm{N}$ Engl J Med. 2005;353(23):2462-2476.

- A clinical trial concerning the efficacy of infliximab in UC.

3. Sandborn WJ, Van Assche G, Reinisch W, et al. Adalimumab induces and maintains clinical remission in patients with moderate-tosevere ulcerative colitis. Gastroenterology. 2012;142(2):257-65 e1-3.

- A clinical trial concerning the efficacy of adalimumab in UC.

4. Reinisch W, Sandborn WJ, Hommes DW, et al. Adalimumab for induction of clinical remission in moderately to severely active ulcerative colitis: results of a randomised controlled trial. Gut. 2011;60(6):780-787.

5. Sandborn WJ, Feagan BG, Marano C, et al. Subcutaneous golimumab induces clinical response and remission in patients with moderate-to-severe ulcerative colitis. Gastroenterology. 2014;146(1):8595. quiz e14-5.

. A clinical trial concerning the efficacy of golimumab in the induction phase of UC.

6. Sandborn WJ, Feagan BG, Marano C, et al. Subcutaneous golimumab maintains clinical response in patients with moderate-tosevere ulcerative colitis. Gastroenterology. 2014;146(1):96-109 e1.

- A clinical trial concerning the efficacy of golimumab in the maintenance phase of UC.

7. Feagan BG, Rutgeerts $P$, Sands BE, et al. Vedolizumab as induction and maintenance therapy for ulcerative colitis. $\mathrm{N}$ Engl J Med. 2013;369(8):699-710.

- A clinical trial concerning the efficacy of vedolizumab in UC

8. Coskun $\mathrm{M}$, Vermeire $\mathrm{S}$, Nielsen $\mathrm{OH}$. Novel targeted therapies for inflammatory bowel disease. Trends Pharmacol Sci. 2017;38(2):127-142.

9. Shealy DJ, Cai A, Staquet K, et al. Characterization of golimumab, a human monoclonal antibody specific for human tumor necrosis factor alpha. MAbs. 2010;2(4):428-439.

10. European Medicines Agency. Pharmacokinetic properties. Available from: http://www.ema.europa.eu/docs/en_GB/document_library/ EPAR_-Product_Information/human/000992/WC500052368.pdf

11. European Medicines Agency. Pharmacological properties. Immunogenicity. Available from: http://www.ema.europa.eu/docs/ en_GB/document_library/EPAR_-Product_Information/human/ 000992/WC500052368.pdf

12. D'Haens G, Sandborn WJ, Feagan BG, et al. A review of activity indices and efficacy end points for clinical trials of medical therapy in adults with ulcerative colitis. Gastroenterology. 2007;132(2):763786.

13. Rutgeerts $P$, Feagan BG, Marano CW, et al. Randomised clinical trial: a placebo-controlled study of intravenous golimumab induction therapy for ulcerative colitis. Aliment Pharmacol Ther. 2015;42 (5):504-514.

14. Colombel JF, Reinisch W, Gibson P, et al. Delayed response to golimumab therapy: UC patient characteristics and long-term clinical outcome-post hoc analyses from the PURSUIT programme. Abstract DOP048. Available from: https://www.ecco-ibd.eu/index. php/publications/congress-abstract-s/abstracts-2016/item/dop048delayed-response-to-golimumab-therapy-uc-patient-characteris tics-and-long-term-clinical-outcomex2014post-hoc-analyses-fromthe-pursuit-programme.html

15. Gibson PR, Feagan BG, Sandborn WJ, et al. Maintenance of efficacy and continuing safety of golimumab for active ulcerative colitis: PURSUIT-SC maintenance study extension through 1 year. Clin Transl Gastroenterol. 2016;7:e168.

16. Brahmania M, Bernstein CN. Physician global assessments or blood tests do not predict mucosal healing in ulcerative colitis. Can J Gastroenterol Hepatol. 2014;28(6):325-329.

17. Singh S, Garg SK, Wang Z, et al. Letter: comparative efficacy of biological therapy in patients with ulcerative colitis. Aliment Pharmacol Ther. 2014;39(12):1432-1433.

18. Stidham RW, Lee TC, Higgins PD, et al. Systematic review with network meta-analysis: the efficacy of anti-tumour necrosis factoralpha agents for the treatment of ulcerative colitis. Aliment Pharmacol Ther. 2014;39(7):660-671.

19. Danese S, Fiorino G, Peyrin-Biroulet $L$, et al. Biological agents for moderately to severely active ulcerative colitis: a systematic review and network meta-analysis. Ann Intern Med. 2014;160(10):704-711.

20. Adedokun OJ, Xu Z, Marano CW, et al. Pharmacokinetics and exposure-response relationship of golimumab in patients with moderately-to-severely active ulcerative colitis: results from phase 2/3 PURSUIT induction and maintenance studies. J Crohns Colitis. 2017;11(1):35-46.

21. Detrez I, Dreesen E, Van Stappen T, et al. Variability in golimumab exposure: a 'Real-Life' observational study in active ulcerative colitis. J Crohns Colitis. 2016;10(5):575-581.

22. Drabik A, Sturm A, Blomacher M, et al. Early monitoring of response (MORE) to golimumab therapy based on fecal calprotectin and trough serum levels in patients with ulcerative colitis: a multicenter prospective study. JMIR Res Protoc. 2016;5(2):e124.

23. Kay J, Fleischmann R, Keystone E, et al. Five-year safety data from 5 clinical trials of subcutaneous golimumab in patients with rheumatoid arthritis, psoriatic arthritis, and ankylosing spondylitis. J Rheumatol. 2016;43(12):2120-2130.

24. Markowitz LE, Dunne EF, Saraiya M, et al. Quadrivalent human papillomavirus vaccine: recommendations of the advisory committee on immunization practices (ACIP). MMWR Recomm Rep. 2007;56(RR-2):1-24.

25. Androulakis I, Zavos C, Christopoulos P, et al. Safety of anti-tumor necrosis factor therapy during pregnancy in patients with inflammatory bowel disease. World J Gastroenterol. 2015;21 (47):13205-13211.

26. Weber-Schoendorfer C, Oppermann M, Wacker E, et al. Pregnancy outcome after TNF-alpha inhibitor therapy during the first trimester: a prospective multicentre cohort study. $\mathrm{Br} J \mathrm{Clin}$ Pharmacol. 2015;80(4):727-739.

- A study of the risks of anti-TNF agents in pregnancy.

27. Kawalec P, Pilc A. An indirect comparison of infliximab versus adalimumab or golimumab for active ulcerative colitis. Arch Med Sci. 2016;12(5):1097-1109.

28. Thorlund K, Druyts $E$, Toor K, et al. Comparative efficacy of golimumab, infliximab, and adalimumab for moderately to severely active ulcerative colitis: a network meta-analysis accounting for differences in trial designs. Expert Rev Gastroenterol Hepatol. 2015;9 (5):693-700 
29. Bendtzen K, Ainsworth M, Steenholdt C, et al. Individual medicine in inflammatory bowel disease: monitoring bioavailability, pharmacokinetics and immunogenicity of anti-tumour necrosis factoralpha antibodies. Scand J Gastroenterol. 2009;44(7):774-781.

- Interesting article concerning the pharmacokinetic data in the case of loss of response to anti-TNF agents.

30. Kay J, Matteson EL, Dasgupta B, et al. Golimumab in patients with active rheumatoid arthritis despite treatment with methotrexate: a randomized, double-blind, placebo-controlled, dose-ranging study. Arthritis Rheum. 2008;58(4):964-975.

31. Ungar B, Kopylov U, Engel T, et al. Addition of an immunomodulator can reverse antibody formation and loss of response in patients treated with adalimumab. Aliment Pharmacol Ther. 2017;45(2):276-282.
- Interesting article concerning the role of immunosuppressors in the prevention of antibody formation against TNF antagonist agents.

32. Dulai PS, Siegel CA, Colombel JF, et al. Systematic review: monotherapy with antitumour necrosis factor alpha agents versus combination therapy with an immunosuppressive for IBD. Gut. 2014;63(12):1843-1853.

33. Lichtenstein GR, Rutgeerts $P$, Sandborn WJ, et al. A pooled analysis of infections, malignancy, and mortality in infliximab- and immunomodulator-treated adult patients with inflammatory bowel disease. Am J Gastroenterol. 2012;107(7):1051-1063.

34. Lichtenstein GR, Diamond $\mathrm{RH}$, Wagner $\mathrm{CL}$, et al. Clinical trial: benefits and risks of immunomodulators and maintenance infliximab for IBD-subgroup analyses across four randomized trials. Aliment Pharmacol Ther. 2009;30(3):210-226. 